\title{
Polymorphism FXII 46C>T and cardiovascular risk: additional data from Spanish and Tunisian patients Georgios Athanasiadis' ${ }^{1}$, Esther Esteban ${ }^{1}$, Magdanela Gayà Vidal ${ }^{1}$, Robert Carreras Torres ${ }^{1}$, Raoudha Bahri² ${ }^{2}$ and Pedro Moral*1
}

\author{
Address: ${ }^{1}$ Unitat d'Antropologia, Facultat de Biologia, Universitat de Barcelona, Barcelona, Spain and ${ }^{2}$ Faculté de Pharmacie de Monastir, \\ Université de Monastir, Monastir, Tunisia \\ Email: Georgios Athanasiadis - athanasiadis@ub.edu; Esther Esteban - mesteban@ub.edu; Magdanela Gayà Vidal - magdagaya@ub.edu; \\ Robert Carreras Torres - robertcarrerastorres@hotmail.com; Raoudha Bahri - bahri@yahoo.fr; Pedro Moral* - pmoral@ub.edu \\ * Corresponding author
}

Published: 31 July 2009

BMC Research Notes 2009, 2:154 doi:10.1 186/1756-0500-2-154

This article is available from: http://www.biomedcentral.com//756-0500/2/154

(C) 2009 Moral et al; licensee BioMed Central Ltd.

This is an Open Access article distributed under the terms of the Creative Commons Attribution License (http://creativecommons.org/licenses/by/2.0), which permits unrestricted use, distribution, and reproduction in any medium, provided the original work is properly cited.
Received: 30 May 2009

Accepted: 31 July 2009

\begin{abstract}
Background: Previous studies showed an association between Coagulation Factor XII 46C $>\mathrm{T}$ polymorphism and variation in FXII plasma levels, as $46 \mathrm{C}>\mathrm{T}$ seems to affect the translation efficiency. Case-control studies in Spanish samples indicated that genotype T/T is an independent risk factor for venous thrombosis, ischemic stroke and acute coronary artery disease. In this study, we tried to reaffirm the importance of $46 \mathrm{C}>\mathrm{T}$ in two samples from Spain and Tunisia.

Findings: A Transmission Disequilibrium Test (TDT) based on I0I family trios from Barcelona with one offspring affected by ischemic heart disease and a classical case-control study based on 76 patients with IHD and 118 healthy individuals from North and Centre-South Tunisia were conducted. Subjects were genotyped for 46C>T and data were analyzed accordingly, revealing no association in any of the two samples (TDT: $P=0.16$, relative risk 1.17 ; case-control study: $P=$ 0.59 , odds ratio 1.36 ).

Conclusion: The results suggest that $46 \mathrm{C}>\mathrm{T}$ is not a risk factor for ischemic heart disease in any of the two analyzed samples and therefore the polymorphism seems not to be a universal risk factor for cardiovascular diseases.
\end{abstract}

\section{Findings}

The gene of coagulation Factor (F) XVII has been repeatedly analysed in many epidemiological studies on cardiovascular (CV) genetic risk with controversial results. It has been reported that FXII plasma levels exhibit a $67 \%$ of heritability and that these levels are strongly determined by F12 gene variants.[1] Although low FXII plasma levels have been reported to affect the development of $\mathrm{CV}$ diseases, it has been recently proposed that, rather than the cause of thrombosis, these low levels are actually the result of it. $[2,3]$

A highlighted F12 polymorphism is the $46 \mathrm{C}>\mathrm{T}$ transition. It seems that $46 \mathrm{C}>\mathrm{T}$ affects the translation efficiency, leading to reduced FXII plasma levels.[4] This polymorphism (with allele frequencies $0.8 / 0.2$ in Caucasians) accounts for $40 \%$ of the variance in FVII activity in a Spanish Mediterranean population and seems to fit a log additive 
model of inheritance with an allele dose-dependent effect. $[1,4]$

Previous genetic association studies between $46 \mathrm{C}>\mathrm{T}$ and $\mathrm{CV}$ risk showed that genotype $\mathrm{T} / \mathrm{T}$ increases significantly the risk for venous thrombosis, ischemic stroke and acute coronary artery disease in a Spanish population. [5-7] The odds ratio in acute coronary artery disease was estimated around 4.8.[7] Conversely, there are also studies that either do not detect genetic association between this genotype and $\mathrm{CV}$ risk or alternatively report association between the $\mathrm{C} / \mathrm{C}$ genotype and CV risk. $[2,8]$

In this context, this study provides additional data about the allele frequency distribution of $46 \mathrm{C}>\mathrm{T}$ in two new Mediterranean samples. These data are used in an attempt to confirm previous genetic association studies, by a double epidemiological design: a family-based analysis (Transmission Disequilibrium Test TDT) applied in a Spanish sample and a classical case-control study applied in a Tunisian sample.

A total of 101 nuclear families $(n=302)$ with one offspring (93 males and 8 females) clinically diagnosed for ischemic heart disease (IHD) were analyzed, all from the area of Barcelona, Spain. A comprehensive description of the families, diagnosis criteria and DNA extraction methods has been reported elsewhere.[9] Moreover, 76 patients with IHD complicated by myocardial infarction, all from Monastir, Tunisia, as well as 118 unrelated healthy autochthonous individuals from North and Central-North Tunisia were also analyzed in an unmatched case-control design.[10] This study has been performed in accordance with the Ethical Committee guidelines of the participating Hospitals and Universities and all subjects participating in the study signed a written informed consent.

Polymorphism 46C>T was genotyped by Real-Time PCR, using the standard TaqMan ${ }^{\circledast}$ SNP genotyping assay protocol of Applied Biosystems for a total volume of $5 \mu \mathrm{l}$ per well. DNA amplification as well as fluorescence intensity measurements of the final reaction product and data col- lection were carried out with an ABI PRISM ${ }^{\circledast} 7900$ HT Sequence Detection System.

Allele frequencies in the Spanish IHD patients and their parents as well as in patients and controls from Tunisia were calculated directly. A TDT analysis was performed in the IHD families from Barcelona, using the FBAT v2.0.2c program.[11] In the Tunisian case-control study, association was examined with Epi Info ${ }^{\mathrm{TM}}$ v3.4.3.[12] This program was also used for the calculation of the relative risk in the TDT analysis and the odds ratio (T/T vs. C/C and C/ $\mathrm{T}$ ) in the case-control design. Sample power was calculated using QUANTO v1.2.3 assuming a CV disease prevalence of 0.07.[13]

Frequencies of the T allele in the Spanish and Tunisian samples are shown in Table 1. In the Spanish families, the TDT analysis indicated a low relative risk of 1.17 (95\% CI: $0.75<\mathrm{RR}<1.82$ ) for the $\mathrm{T}$ allele, without any significant transmission to the patients $(\mathrm{p}=0.16)$. Power calculations revealed that the minimum detectable risk our family sample size $(\mathrm{n}=101)$ could find was approximately 1.9 (with a power $>80 \%$ ). In the Tunisian sample, the estimated odds ratio for the T/T genotype was 1.36 (95\% CI: $0.36<$ OR $<4.93$ ), without any significant association ( $\mathrm{p}$ $=0.59$ ). Again, the minimum detectable effect our set of 76 patients and 118 controls could find was around 1.9. Finally, no significant differences were found in any pairwise comparison of the data in Table 1.

Our study showed that the allele frequencies of the FXII $46 \mathrm{C}>\mathrm{T}$ polymorphism are similar in the Spanish and Tunisian samples, in accordance with the Caucasian pattern. This suggests a stable presence of $46 \mathrm{C}>\mathrm{T}$ in different Mediterranean regions. On the other hand, our results indicate the absence of association between $46 \mathrm{C}>\mathrm{T}$ and CV disease in both samples.

The similar allele frequency distributions between Spanish patients and parents and between Tunisian patients and controls provide a first rough estimate of the lack of association. Moreover, our Tunisian sample has enough power ( $>99 \%)$ to detect an odds ratio of 4.8 , as reported

Table I: Allele and genotype frequencies of the $46 \mathrm{C}>\mathrm{T}$ in the Spanish and Tunisian CV patients

\begin{tabular}{|c|c|c|c|c|c|c|c|}
\hline & & \multicolumn{2}{|c|}{ Allele frequencies } & \multicolumn{3}{|c|}{ Genotype frequencies } & \multirow[b]{2}{*}{$\mathrm{N}$} \\
\hline & & $\mathrm{C}$ & $\mathrm{T}$ & $\mathrm{CC}$ & $\mathrm{CT}$ & TT & \\
\hline \multirow[t]{2}{*}{ Spain } & affected children & 0.768 & 0.232 & 0.590 & 0.356 & 0.054 & 97 \\
\hline & parents & 0.799 & 0.201 & 0.638 & 0.321 & 0.040 & 137 \\
\hline \multirow[t]{2}{*}{ Tunisia } & cases & 0.770 & 0.230 & 0.593 & 0.354 & 0.053 & 76 \\
\hline & controls & 0.750 & 0.250 & 0.563 & 0.375 & 0.063 & 118 \\
\hline
\end{tabular}


elsewhere, reinforcing the consistency of the lack of association detected.[7] As far as we know, this is the first time the potential role of $46 \mathrm{C}>\mathrm{T}$ is tested through a familybased TDT analysis. Out findings confirm the negative results of the case-control design, always taking into account that our family trios have power only to detect risks above 1.9.

The diverse results of previous studies regarding association between polymorphism $46 \mathrm{C}>\mathrm{T}$ and $\mathrm{CV}$ disease could be explained, at least in part, by the differences in study design, the definition of inclusion and exclusion criteria and the number of participants. In our case, the low sample size in the TDT study and the lack of a matched casecontrol design impose some caution in the interpretation of our results.

In short, our results provide indirect evidence that $46 \mathrm{C}>\mathrm{T}$ is not a universal independent risk factor for CV diseases. What seems to be well established is that the $\mathrm{T}$ allele affects the variation of intermediate phenotypes (FXII plasma levels). If this is true, then the diversity of results in genetic association studies is hard to explain. This observation leads us to adopt the idea that the low levels of FXII are the consequence rather than the cause of the disease, as has been recently suggested.[3] This idea finds solid ground when combined with other independent results on the lack of causality between FXII deficiency and venous thrombosis. [14,15] In this context, 46C>T polymorphism may not be a direct determinant of $\mathrm{CV}$ risk.

\section{Competing interests}

The authors declare that they have no competing interests.

\section{Authors' contributions}

GA carried out the SNP genotyping, participated in the statistical analyses and wrote the original manuscript. EE gave important advice for the improvement of the manuscript. MGV carried out the Spanish sample preparation. RCT helped with the statistical analyses. RB carried out the DNA extraction from the Tunisian samples. PM participated in the design and coordination of the study and helped to draft the manuscript. All authors read and approved the final manuscript.

\section{Acknowledgements}

This research has been supported by the Ministerio de Ciencia y Tecnología CGL2005-339I and Generalitat de Catalunya SGR00252 projects. The work of GA has been financed by an FPU grant from the Ministerio de Ciencia e Innovación (grant reference: AP2005-4425).

\section{References}

I. Soria JM, Almasy L, Souto JC, Bacq D, Buil A, Faure A, MartínezMarchán E, Mateo J, Borrell M, Stone W, Lathrop M, Fontcuberta J, Blangero J: A quantitative trait locus in human factor $X I I$ gene influences both plasma factor XII levels and susceptibility to thrombotic disease. Am J Hum Genet 2002, 70:567-574.

2. Bach J, Endler G, Winkelmann BR, Boehm BO, Maerz W, Mannhalter C, Hellstern P: Coagulation factor XII activity, activated factor XII, distribution of factor XII C46T gene polymorphism and coronary risk. J Thromb Haemost 2008, 6:291-296.

3. Kanaji T: Lower factor XII activity is a risk marker rather than a risk factor for cardiovascular disease: a rebuttal. J Thromb Haemost 2008, 6: 1053-1054.

4. Kanaji T, Okamura T, Osaki K, Kuroiwa M, Shimoda K, Hamasaki N, Niho Y: A common genetic polymorphism (46 C to $\mathrm{T}$ substitution) in the 5 -untranslated region of the coagulation factor XII gene is associated with low translation efficiency and decrease in plasma factor XII level. Blood 1998, $91: 2010-2014$.

5. Tirado I, Soria JM, Mateo J, Oliver A, Souto JC, Santamaria A, Felices $\mathrm{R}$, Borrell $\mathrm{M}$, Fontcuberta J: Association after linkage analysis indicates that homozygosity for the $46 \mathrm{C}-->T$ polymorphism in the $F I 2$ gene is a genetic risk factor for venous thrombosis. Thromb Haemost 2004, 92:892-893.

6. Santamaría A, Mateo J, Tirado I, Oliver A, Belvís R, Martí-Fábregas J, Felices R, Soria JM, Souto JC, Fontcuberta J: Homozygosity of the $T$ allele of the $46 \mathrm{C}->T$ polymorphism in the $F I 2$ gene is a risk factor for ischemic stroke in the Spanish population. Stroke 2004, 35:1795-1799.

7. Santamaría A, Martínez-Rubio A, Mateo J, Tirado I, Soria JM, Fontcuberta J: Homozygosity of the T allele of the $46 \mathrm{C}->-\mathrm{T}$ polymorphism in the FI2 gene is a risk factor for acute coronary artery disease in the Spanish population. Haematologica 2004, 89:878-879.

8. Kanaji T, Watanabe $\mathrm{K}$, Hattori $\mathrm{S}$, Urata M, lida $\mathrm{H}$, Kinoshita $\mathrm{S}$, Kayamori Y, Kang D, Hamasaki N: Factor XII gene (FI2) -4C/C polymorphism in combination with low protein $\mathbf{S}$ activity is associated with deep vein thrombosis. Thromb Haemost 2006, 96:854-855

9. Via M, López-Alomar A, Valveny N, González-Pérez E, Bao M, Esteban $E$, Pintó $X$, Domingo E, Moral P: Lack of association between eNOS gene polymorphisms and ischemic heart disease in the Spanish population. Am J Med Genet A 2003, I I 6:243-248.

10. Bahri R, Esteban E, Moral P, Chaabani H: New insights into the genetic history of Tunisians: Data from Alu insertion and apolipoprotein E gene polymorphisms. Ann Hum Biol 2008, 35:22-33.

II. Laird N, Horvath S, Xu X: Implementing a unified approach to family based tests of association. Genet Epidemiol 2000, 19:36-42.

12. Dean AG, Dean JA, Burton AH, Dicker RC: Epi Info ${ }^{\mathrm{TM}}$ : a general purpose microcomputer program for health information systems. Am J Preventive Medicine 1991, 7:178-182.

13. Gauderman WJ, Morrison JM: QUANTO I.I: A computer program for power and sample size calculations for genetic-epidemiology studies. 2006 [http://hydra.usc.edu/gxe].

14. Girolami A, Randi ML, Gavasso S, Lombardi AM, Spiezia F: The occasional venous thromboses seen in patients with severe (homozygous) FXII deficiency are probably due to associated risk factors: a study of prevalence in 21 patients and review of the literature. J Thromb Thrombolysis 2004, I7:139-143.

15. Lombardi AM, Bortoletto E, Scarparo P, Scapin M, Santarossa L, Girolami A: Genetic study in patients with factor XII deficiency: a report of three new mutations exon I 3 (Q50ISTOP), exon $14(\mathrm{P} 547 \mathrm{~L})$ and $-\mathrm{I} \mathrm{C}>\mathrm{T}$ promoter region in three compound heterozygotes. Blood Coagul Fibrinolysis 2008, 19:639-643. 